\title{
Remaniements sédimentaires superficiels sur l'estran occidental de la baie du Mont-Saint-Michel
}

\author{
C. Bonnot-Courtois ${ }^{1}$, P. Bassoullet ${ }^{2,}{ }^{*}$, B. Tessier ${ }^{3}$, F. Cayocca ${ }^{2}$, P. Le Hir ${ }^{2}$, A. Baltzer ${ }^{3}$ \\ ${ }^{1}$ CNRS, UMR 8586 PRODIG, Laboratoire de Géomorphologie et Environnement littoral. 15, boulevard de la mer, \\ 35800 Dinard \\ ${ }^{2}$ IFREMER, DYNECO/PHYSED. BP 70.29280 Plouzané \\ ${ }^{3}$ CNRS, UMR 6143 M2C, Université de Caen, 14000 Caen \\ *: Corresponding author : Bassoullet P., email address : Philippe.Bassoullet@ifremer.fr
}

\section{Résumé:}

Les rythmes et l'ampleur des processus « érosion-sédimentation » ont été suivis, à trois échelles de temps, sur le vaste estran sablo-vaseux de la partie occidentale de la baie du Mont-Saint-Michel. Les mesures in situ du niveau de l'interface eau-sédiment montrent que l'amplitude générale des remaniements est généralement inférieure à $5 \mathrm{~cm}$ et variable d'ouest en est. L'influence du vent sur les remaniements superficiels souligne des phases d'érosion rapidement suivies par des phases de sédimentation de même amplitude sur l'ensemble de l'estran. Un modèle numérique hydro-sédimentaire, validé par des mesures sur l'estran, permet de simuler les courants et les transferts de sédiments en zone intertidale.

\begin{abstract}
:
In order to define and quantify the rhythm and amplitude of the processes of erosion and sedimentation on broad tidal flats, in situ measurements of sediment elevation have been performed in the western part of the Mont-Saint-Michel bay. The amplitude of reworking is generally low $(< \pm 5 \mathrm{~cm})$, and variable from west to east. The wind influence on tidal currents
\end{abstract}


and surficial reworking emphasizes erosion phases rapidly followed by accretion of similar amplitude on the whole tidal flat. A numerical model for hydrodynamics and sediment transport is applied to Mont Saint Michel Bay, where ecological concerns are related to sediment transfers.

MOTS CLES : Estran sablo-vaseux, Mesures in situ, Bilan érosion-sédimentation, Modèle numérique, Transport sédimentaire.

KEY WORDS : Tidal sandy-mud flat, "In situ" measurements, Erosion-sedimentation budget, Numerical model, Sediment transport

\section{Introduction}

La baie du Mont Saint-Michel est l'une des principales régions de production conchylicole de la façade de la Manche. La diminution de la productivité des installations ostréicoles et mytilicoles a motivé la mise en place, dans le cadre du Programme National Environnement Côtier, d'un chantier intitulé : "Capacité trophique de la baie du Mont Saint-Michel. L’étude de l'environnement vivant est intimement liée à celle de l'environnement physique (hydrodynamique et sédimentaire) au travers de divers paramètres que sont la turbidité de l'eau, la remise en suspension de sédiments, l'évolution de la nature du fond. Le thème hydrosédimentaire du chantier PNEC avait pour objectif de caractériser les transports dissous et particulaires et de mettre en place une modélisation numérique devant constituer la base physique de la modélisation écologique.

L'objectif de cet article est d'analyser les rythmes et l'ampleur des processus érosion - sédimentation qui s'opèrent sur ces très vastes replats de marée où sont implantées les installations conchylicoles. L'étude se base sur les résultats de nombreuses campagnes de mesures in situ qui ont permis de suivre les évolutions de la couverture sédimentaire superficielle, et de mettre en évidence les phases d'érosion et de dépôt en fonction des conditions marégraphiques et météorologiques ainsi que de la localisation sur l'estran (Bonnot-Courtois et al., 2005 et 2006). La modélisation numérique des processus responsables de ces évolutions spatiotemporelles facilite la compréhension globale des phénomènes.

La partie occidentale de la baie du Mont Saint-Michel est caractérisée par un très large estran vaseux à sableux (figure 1), évoluant en régime mégatidal sous l'action permanente des courants de marée (Larsonneur et al. 1989 ; L'Homer et al. 1999). Ces courants de type alternatifs dans la partie centrale de la baie, s'atténuent et prennent un caractère giratoire dans la baie de Cancale. De même, les houles dominantes de NW sont très largement amorties à l'Ouest alors que leur action est significative au centre de la baie. Elle y induit la construction de bancs sablocoquilliers de grande taille dans la partie haute de l'estran (Bonnot-Courtois et al. 2004), et contribue au remaniement général de la surface du tidal flat. 
Afin de mieux caractériser les évolutions du sédiment et les processus de remaniements superficiels, un suivi du niveau du sédiment a été réalisé dans ce vaste tidal flat de la partie occidentale de la baie, à plusieurs échelles de temps.

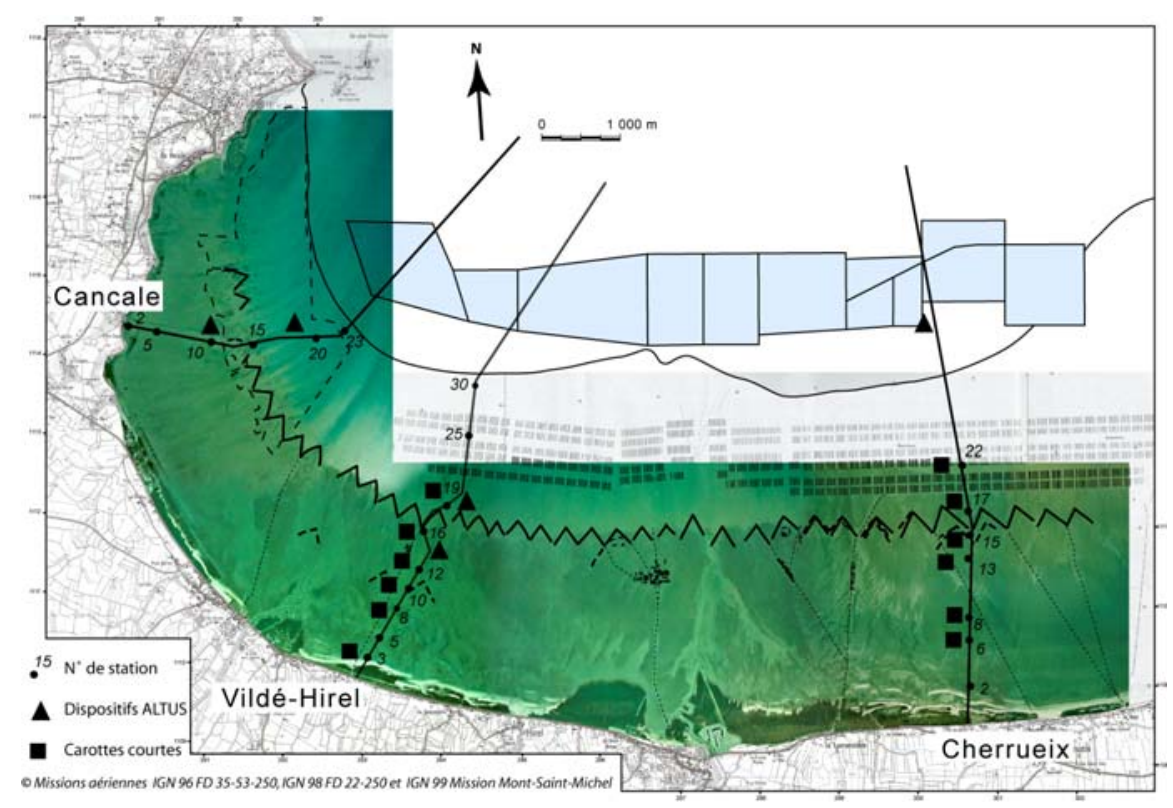

Figure 1. Morphologie de l'estran occidental de la baie du Mont Saint-Michel et implantation des dispositifs du suivi le long des trois radiales. La station SAMPLE a été installée à la station 20 de la radiale de Vildé-Hirel.

\section{Méthodologie}

Pour les évolutions sédimentaires à court terme (cycle tidal journalier), des dispositifs ALTUS (figure 2a) de mesures acoustiques en continu des variations du niveau du sédiment ont été implantés en certaines stations (figure 1) sur plusieurs mois (Jestin et al., 1998). Ces mesures permettent de suivre les rythmes d'érosionsédimentation et de les mettre en relation avec les cycles tidaux et/ou les épisodes de plus forte agitation.

Pour le suivi à moyen terme (cycle lunaire), des dispositifs de type jalons permettant de mesurer le niveau du sédiment (figure 2b) ont été implantés en des stations espacées de $150 \mathrm{~m}$ le long de trois radiales transversales, longues de 3 à $4 \mathrm{~km}$, représentatives des différents environnements sédimentaires de l'estran 
occidental (figure 1). Les relevés ont été effectués en vive-eau, à un rythme mensuel au cours des années 2002 et 2003, et trimestriel à partir de 2004. La précision des mesures est en moyenne de $\pm 3 \mathrm{~mm}$ et varie en fonction de la nature sableuse ou vaseuse du sédiment et du temps d'émersion de la surface de l'estran.
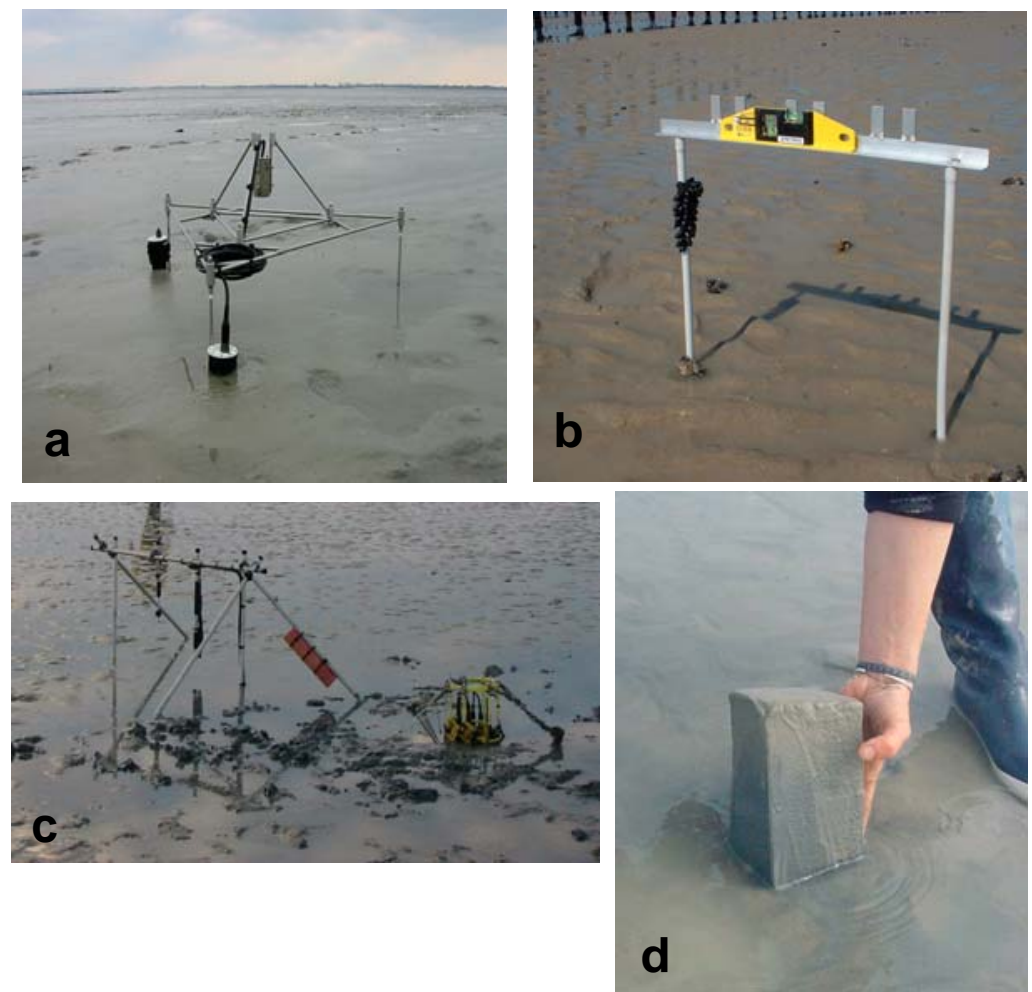

Figure 2 . Dispositif ALTUS (a), stations d'implantation de jalons pour le suivi moyen terme du niveau du sédiment (b), station SAMPLE (c) et mini-carottages (d) (sur $20 \mathrm{~cm}$ ) sur l'estran.

Pour les évolutions à long terme (échelle pluri-annuelle) des carottes courtes (20 cm à $30 \mathrm{~cm}$, figure 2c) ont été prélevées en certaines stations le long des radiales (figure 1) afin de caractériser les faciès sédimentaires associés aux processus de remaniement.

Les mesures de courant disponibles sur la baie (Migniot, 1998) ont généralement été réalisées au large et sur un seul cycle de marée, et aucune n'a été effectuée sur l'estran. De nouvelles campagnes de mesures long terme ont été menées en 2003, en zone intertidale par courantomètre électromagnétique à $30 \mathrm{~cm}$ de l'interface eausédiment, (station multiparamètres SAMPLE) (figure 2) (Jestin et al., 1994). 


\section{Cadre morpho-sédimentaire}

Les trois radiales suivies correspondent à différents environnements sédimentaires (Caline, 1982 ; Bonnot-Courtois et al., 2002), auxquels correspondent des faciès allant des vases pures aux sables propres dépourvus de fraction fine (figure 3).
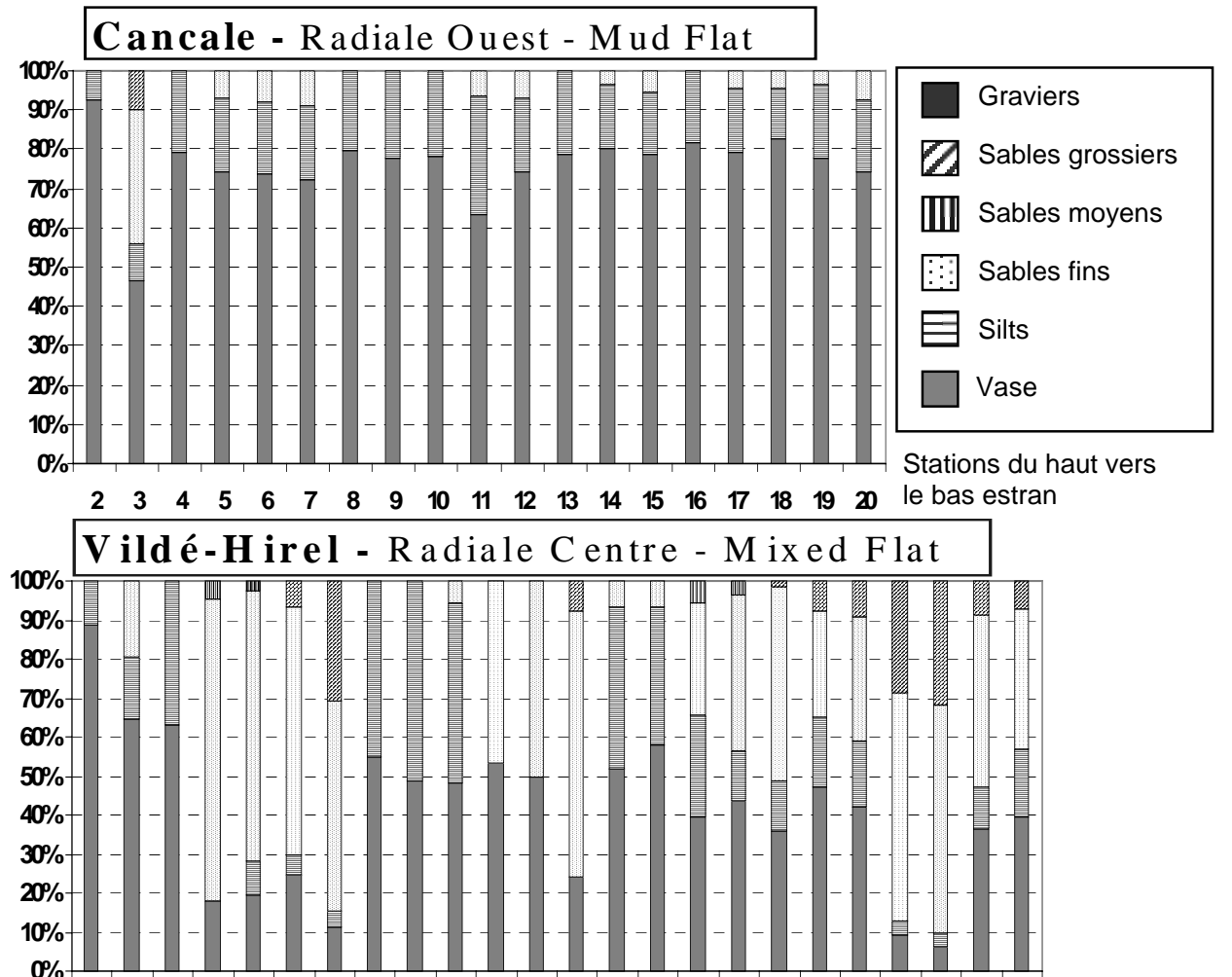

$\begin{array}{lllllllllllllllllllllllll}2 & 3 & 4 & 5 & 6 & 7 & 8 & 9 & 10 & 11 & 12 & 13 & 14 & 15 & 16 & 17 & 18 & 19 & 20 & 21 & 22 & 23 & 24 & 25 & \text { Stations }\end{array}$

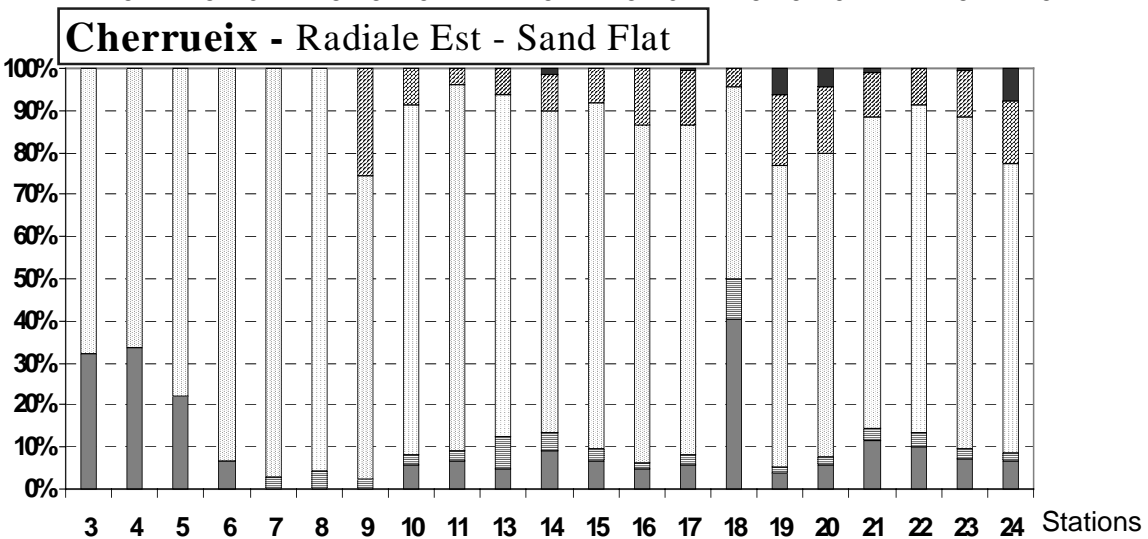

Figure 3 . Evolution granulométrique du sédiment superficiel du haut estran (stations 2-3) vers le bas estran (Stations 20-25), le lona des trois radiales. 
Le gradient granulométrique augmente d' Ouest en Est, les dépôts sédimentaires correspondant successivement à :

- un mud flat en baie de Cancale, constitué essentiellement de vases fines $(<40 \mu \mathrm{m})$ peu consolidées (figure 3). La partie moyenne et basse de l'estran est occupée par des installations ostréicoles. La pente générale de l'estran est de 2,5 \%o ;

- un tidal flat sablo-vaseux dans le secteur de Vildé-Hirel (figure 3) où des vasières occupent la partie médiane de l'estran et passent progressivement à des faciès plus sableux dans la partie basse de l'estran, occupée par ailleurs par des anciennes pêcheries et par des installations mytilicoles. La pente du moyen estran est de 3,1\%o, celle du bas estran, plus faible, de $2 \%$;

- un sand flat au centre de la baie à Cherrueix, dominé par des faciès de sables fins tout le long de la radiale (figure 3). Les pêcheries et les lignes de bouchots occupent l'ensemble du bas estran. La pente générale de l'estran est de 3,4 \%.

\section{Résultats}

\subsection{Les évolutions sédimentaires à court terme}

L'exploitation des enregistrements ALTUS a permis de suivre l'évolution du sédiment superficiel et de détecter une éventuelle "couche crème" temporaire. La cadence d'acquisition, l'enregistrement simultané de la hauteur d'eau et les corrélations avec les conditions météorologiques (vitesse et direction des vents) permettent la mise en évidence des périodes pendant lesquelles se produisent les remaniements ou les dépôts. Une quantification est ainsi possible selon la nature du forçage en jeu, la précision des mesures étant de l’ordre de $\pm 2 \mathrm{~mm}$.

Pour la radiale Vildé-Hirel, les variations notables du niveau de sédiment concernent le secteur situé en amont des pêcheries (figure 4). L'enregistrement de l'altimètre relève en 2 jours des érosions de 5,5 cm lors d'épisodes de vents de secteur Sud. Ce phénomène est renforcé s'il est conjugué à des marées de fortes amplitudes. Les vents de secteur Est fournissent, durant une semaine, de fortes charges en suspension. La recharge de l'estran est continue fin mars - début avril seulement perturbée par un coup de vent le 02 avril 2003, décapant $3 \mathrm{~cm}$.

L'enregistrement altimétrique sur l'estran médian de la radiale Cancale (figure 4) permet de quantifier $(2 \mathrm{~cm}$ ) le dépôt d'une couche de vase sous l'action de vents d'Est durant 6 jours (mi-mars 2003). Cet enregistrement permet également d'écarter l'action tidale dans ce transit sédimentaire. 

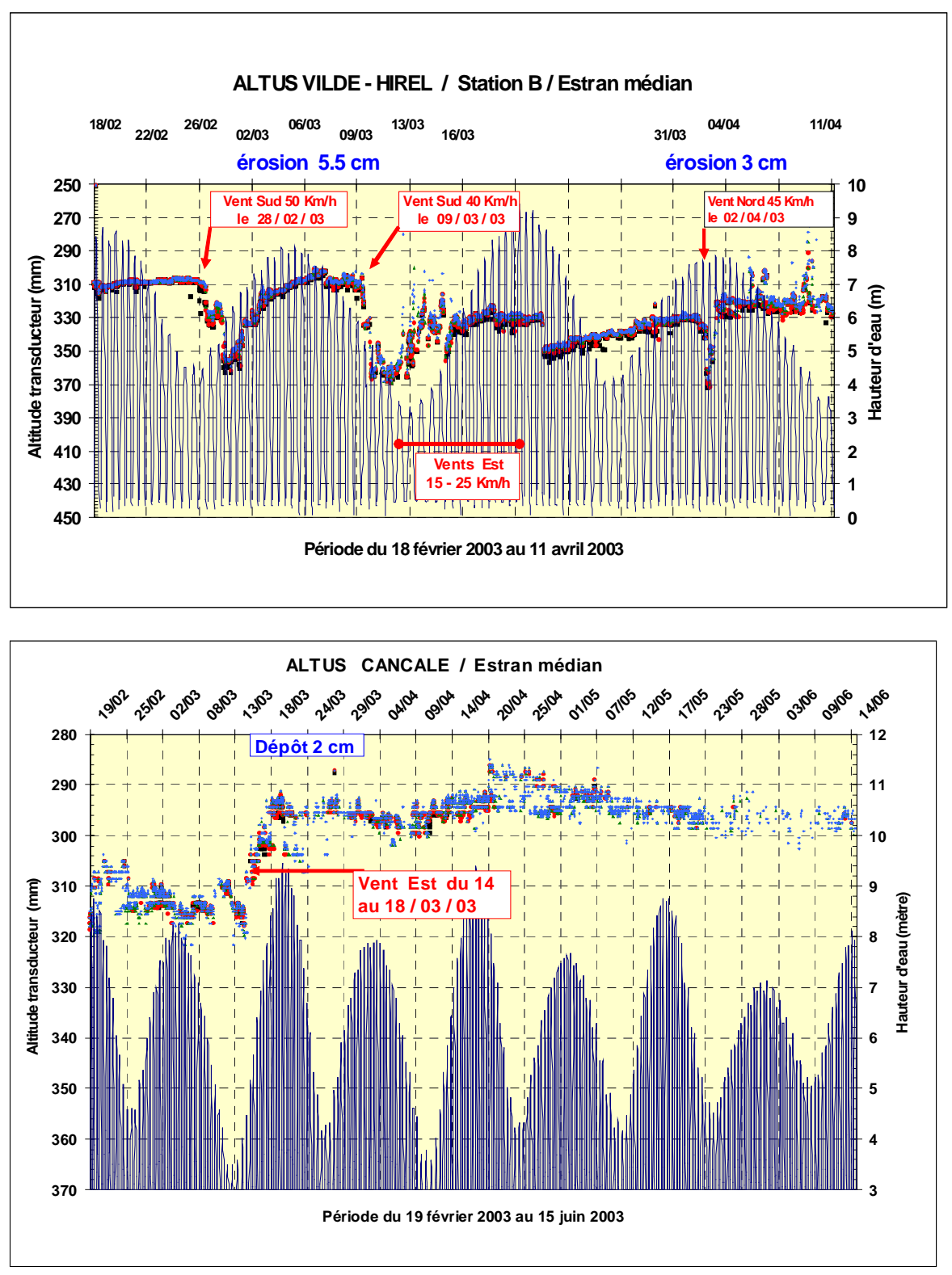

Figure 4 . Suivis ALTUS en amont des installations ostréicoles et des pêcheries au niveau de l'estran médian de Vildé-Hirel et de Cancale [les différentes teintes du niveau sédimentaire correspondent à diverses interfaces caractérisant des degrés de compaction du sédiment] 


\subsection{Les évolutions à moyen terme : le bilan "érosion-sédimentation" le long des trois radiales}

Les résultats du suivi depuis 2002 (Bonnot-Courtois et al. 2006), synthétisés sur la figure 5, montrent que l'amplitude des variations de niveau du sédiment est faible, généralement inférieure à $5 \mathrm{~cm})$. Des variations de plus forte amplitude $(10 \mathrm{~cm})$ ont été mesurées soit localement au niveau du haut estran de Vildé-Hirel et/ou à certaines périodes sur l'ensemble de l'estran, par exemple à Cancale au printemps 2004.

Les remaniements sont de l'ordre de $\pm 5 \mathrm{~cm}$ à Cancale et à Vildé-Hirel, mais inférieurs à $\pm 2 \mathrm{~cm}$ à Cherrueix. Une phase d'érosion des vases superficielles à Vildé-Hirel correspond à une phase de sédimentation à Cancale (entre novembre et décembre 2002). Conformément aux observations de terrain, ceci semble indiquer un équilibre spatial entre les deux radiales les plus occidentales.

Le bilan érosion - sédimentation est à l'équilibre au cours du temps :

- à l'échelle saisonnière : en période hivernale, des phases d'érosion de plusieurs $\mathrm{cm}$ se produisent sur l'ensemble de l'estran mais sont rapidement suivies par des phases de sédimentation de même amplitude (entre mars et septembre 2004 à Cancale).

- - à l'échelle pluri-annuelle, sur toute la période du suivi (3 ans).

Enfin, quelle que soit la radiale considérée, y compris celle de Cancale, il n 'y a pas de relation directe entre l'amplitude des remaniements et la cote altimétrique de l'estran.

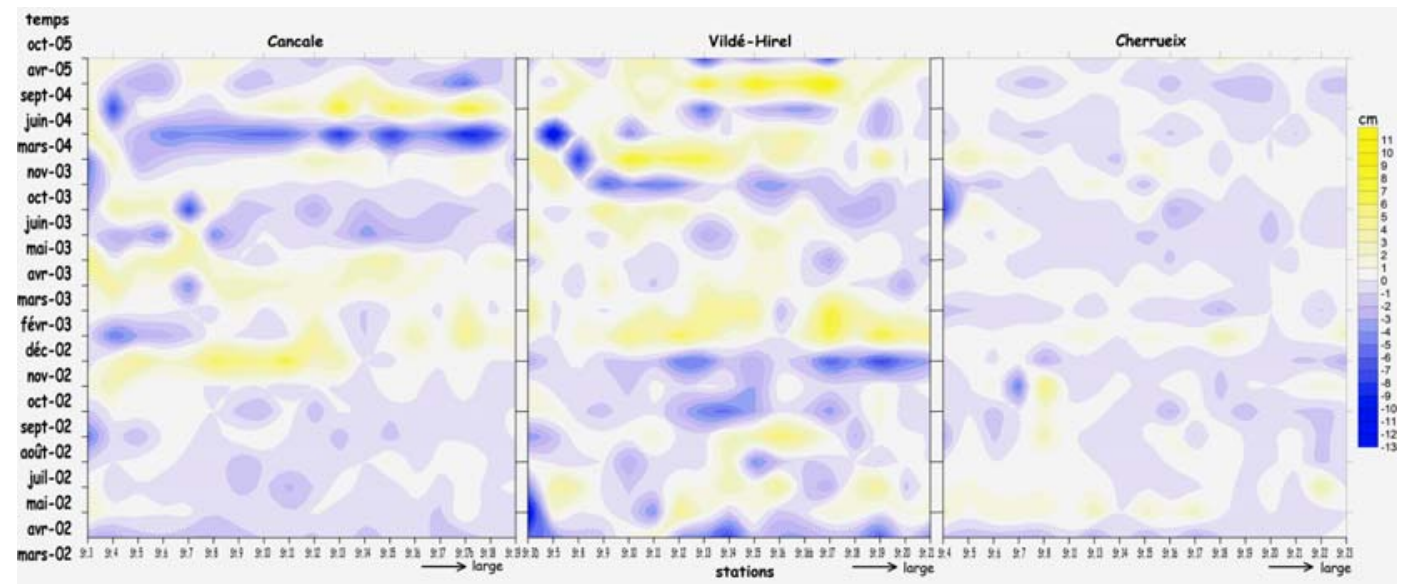

Figure 5 . Remaniements sédimentaires le long des trois radiales entre 2002 et 2005. Echelle des couleurs : Bleu - érosion, Blanc - stabilité, Jaune - sédimentation [en abscisse : succession des stations du haut vers le bas estran, en ordonnée : date des relevés] 


\subsection{Les évolutions à long terme : enregistrements sédimentaires des processus}

Seules les radiales de Vildé-Hirel et de Cherrueix ont fait l'objet de prélèvement de carottes courtes pour l'analyse des successions sédimentaires qui montrent (figure 6) :

- une assez faible variabilité générale de faciès, en accord avec la répartition des sédiments superficiels, représentés par des sables coquilliers, des sables fins à moyens et des vases (Canuet, 2003);

- la préservation des drapages de vases est meilleure à Vildé-Hirel qu’à Cherrueix, soulignant que l'énergie est globalement plus faible vers l'Ouest ;

à Vildé-Hirel, la préservation des drapages est meilleure dans la partie moyenne et basse de l'estran que dans la partie haute soumise aux remaniements par les houles.

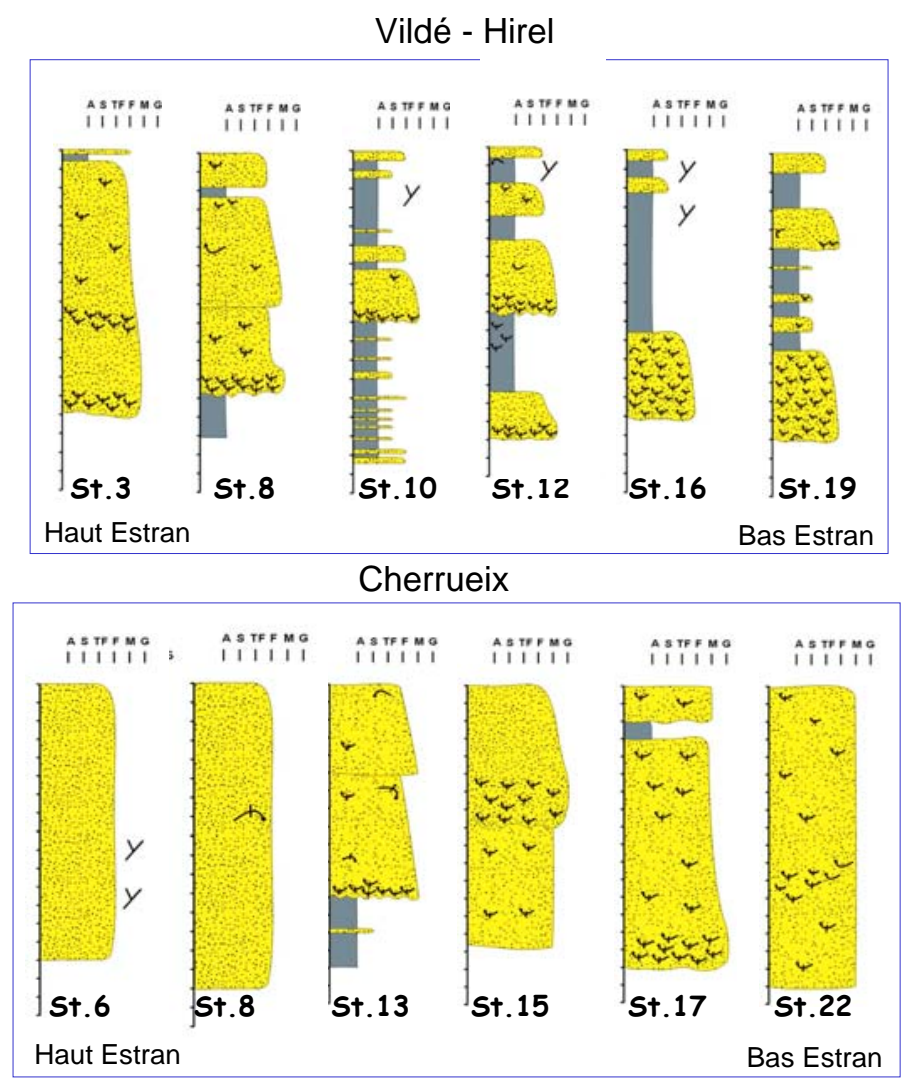

Figure 6 . Enregistrements sédimentaires le long des radiales de Vildé-Hirel et Cherrueix. 


\section{Influence du vent sur les remaniements superficiels}

Plusieurs modèles hydrodynamiques ont décrit les régimes de courants en Baie du Mont Saint Michel (Ehrhold, 1999). Les caractéristiques principales concernent les fortes vitesses rencontrées devant la pointe du Grouin et dans les chenaux du Sud-Est de la Baie. Le modèle hydro-sédimentaire SiAM3D (Le Hir \& Cayocca, 2002) a été utilisé dans sa version bidimensionnelle (coordonnées sigma, une seule couche) en raison du marnage exceptionnel de cette zone (jusqu'à 14 m en vive eau) qui est responsable d'un intense mélange sur la verticale. La bathymétrie a été construite à partir des sources les plus récentes disponibles sur la zone, c'est-à-dire: - un levé LIDAR de 2003, dont la résolution horizontale est de 1m ;

- un levé IFREMER de 2003, effectué par sondeur mono-faisceau entre - 4m et - 22m (IGN69, profils espacés de 200m) ;

- les cartes du SHOM pour les zones non couvertes par les levés ci-dessus.

La résolution spatiale du maillage (cartésien irrégulier) varie de 200 à 500 m et les conditions aux limites du large du modèle sont fournies en hauteur d'eau, et sont calculées à partir de 115 harmoniques de marée (Le Roy et Simon, 2003). Les débits des 3 fleuves principaux (Couesnon, Sée, Sélune) sont prescrits. Le vent est considéré uniforme sur le domaine de simulation, et provient d'un point de sortie du modèle Aladin (Météo France) situé au milieu de la Baie du Mont Saint Michel. L' écart de norme entre le vent résultant de l'analyse Aladin et le vent mesuré à la station météorologique de Dinard (située à $16 \mathrm{~km}$ à l'Ouest de Cancale) est de l’ordre de 20\%, et est indépendant de la direction (Cayocca et al., 2006)

Les mesures de courant présentées ici concernent les résultats obtenus à la station SAMPLE implantée sur le bas estran de Vildé-Hirel en mars 2003. La comparaison des mesures avec le modèle se fait par intégration sur la verticale dans le cas des mesures en profil, et en supposant un profil logarithmique des vitesses pour la mesure SAMPLE. Il existe une bonne concordance entre les mesures in situ et les résultats du modèle (figure 7).

Les résultats montrent une très forte variabilité en morte eau (figure $7 A$ ), qui peut en partie être expliquée par le vent. En particulier, les vents de secteur Ouest induisent une composante zonale des vitesses vers l'Est (11 mars) et inversement les vents de secteur Est (14 Mars) déportent les roses de courants nettement vers l'Ouest. Par plus forts coefficients (10 Mars et 15 Mars), le caractère alternatif se renforce sans différence entre des vents de SW (le 10) et des vents d'Est (le 15). 


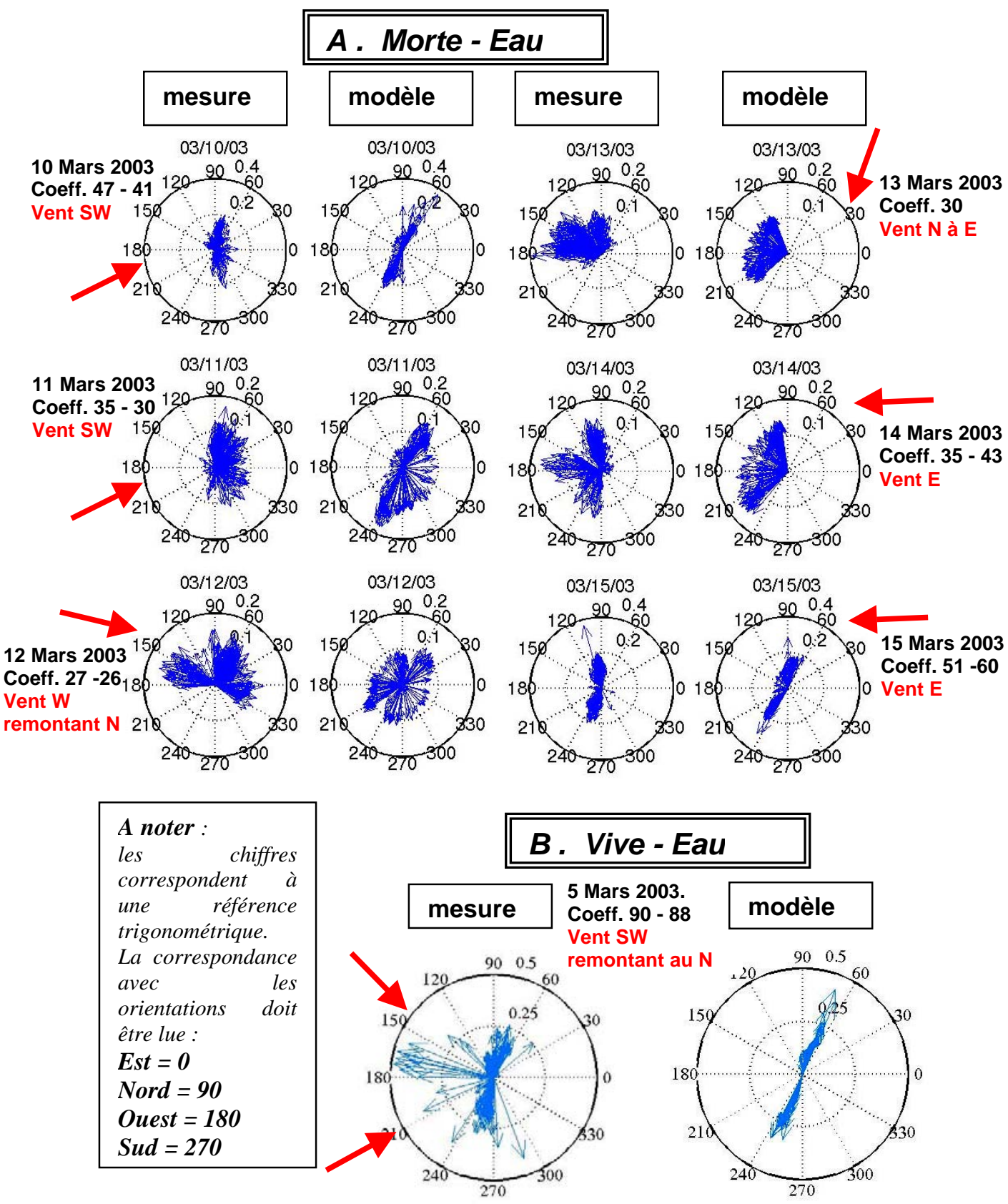

Figure 7 : Courants mesurés par la station SAMPLE et simulés par le modèle.

A : en morte eau (du 10 au 15 mars 2003) B : en vive-eau (5 Mars 2003).

Vitesses de courants indiquées en m.s ${ }^{-1}$. Intensités des vents de 6 à 10 m.s. 
L'effet du vent de Nord ou de Sud est moins clair. Par exemple le 12 mars, on observe la composante méridienne du courant dirigée vers le Nord tout au long de la marée par un vent remontant vers le Nord. Le modèle représente bien cette forte variabilité, et les séries temporelles (non présentées ici) montrent que l'amplitude des vitesses est bien simulée. En vive eau (figure 7B) le vent n'a plus d'influence sur l'orientation des courants. La direction du courant ne présente pas la forte variabilité observée en morte eau, et les courants sont alternatifs. La dispersion observée pour les courants mesurés à la station Sample ne se retrouve pas sur la rose des courants déduite du modèle, mais le caractère alternatif reste bien marqué.

La comparaison entre les rythmes d'érosion - sédimentation et les données de vents à Dinard sur la période mars 2002 à juin 2003 montre :

* que les épisodes de vents les plus forts correspondent aux plus fortes amplitudes de remaniements, qu'il s'agisse de sédimentation ou d'érosion,

* une très grande variabilité des réponses de l'estran aux épisodes de vents forts quelle qu'en soit leur direction. En effet, il n'existe pas de relation univoque entre la direction des vents et les rythmes d'érosion ou de sédimentation sur les deux radiales les plus occidentales.

Cependant, les mesures altimétriques de terrain effectuées au niveau des trois radiales suivies montrent les tendances suivantes :

* A Cancale, des Vents d'Est - Sud-Est sur plusieurs jours en vive-eau correspondent à un dépôt de vase fine très liquide de 2 à $10 \mathrm{~cm}$, en corrélation avec un décapage de 1 à $4 \mathrm{~cm}$ des sédiments superficiels à Hirel et Cherrueix sur la même période. Des vents de secteurs Nord et Ouest, continus sur plusieurs jours sans vent d'Est, conduisent à une érosion globale de l'estran de 2 à $3 \mathrm{~cm}$ à Cancale. A la même période, le moyen estran de Cherrueix comprend des placages de vase fine.

Sur l'estran de Vildé-Hirel, des érosions de plusieurs $\mathrm{cm}$ (jusqu'à $5 \mathrm{~cm}$ ) ont été observées par vents de Sud conjugués avec des marées de vive-eau. Des vents de secteurs Nord à Ouest conduisent à un rechargement de cet estran dans les mêmes proportions. Par ailleurs, sur le haut estran, une érosion de $22 \mathrm{~cm}$ en 10 mois des anciennes vasières indurées a été mesurée en avant des cordons coquilliers, avec décapage par blocs produisant des galets mous classiques dans de tels faciès.

Sur la radiale de Cherrueix qui comporte des faciès plus sableux, des érosions de plus faible amplitude (de 1 à $4 \mathrm{~cm}$ ) de l'ensemble de l'estran ont été constatées notamment par vents de secteurs Sud à Sud-Est. A l'inverse, des vents de secteurs Ouest à Nord-Ouest sont associés à des dépôts de fines, en particulier dans les creux de rides. 
En résumé, l'action des vents sur les différents secteurs d'estran étudiés peut être schématisée par la figure 8. Ainsi, l'action érosive des vents de secteurs Ouest à Nord sur l'ensemble de l'estran de Cancale peut être mise en relation avec le dépôt de sédiments fins, par ces mêmes vents, sur le secteur amont des pêcheries de l'estran de Vildé-Hirel. De même , les vents de Sud-Est érodent le moyen estran de Vildé-Hirel et "engraissent" le mud flat de Cancale.

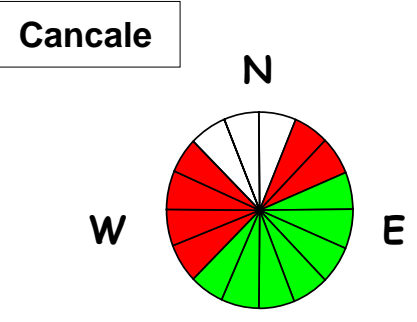

S

Amont des tables ostréicoles

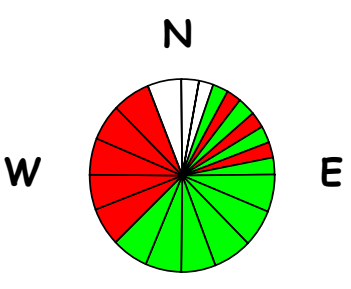

S

Bas-estran

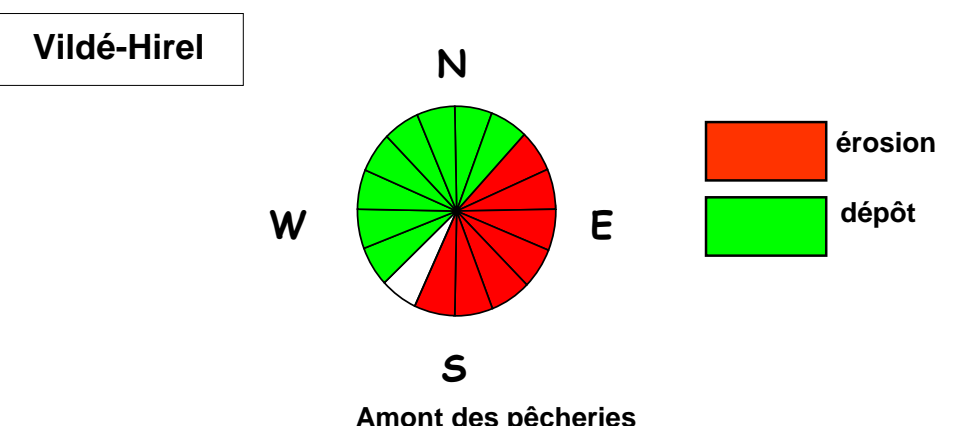

Figure 8 : Synthèse de l'influence du vent sur les remaniements sédimentaires superficiels pour les deux radiales les plus occidentales.

\section{Conclusion}

Le suivi des processus "érosion-sédimentation" réalisé à plusieurs échelles de temps a permis de détailler la dynamique des sédiments superficiels de l'estran occidental de la baie du Mont Saint-Michel. 
Les mesures à court terme (type ALTUS) qui permettent souvent d'identifier et de discerner les forçages océano-météo et d'en quantifier les effets sur le sédiment superficiel sont directement complémentaires des mesures à moyen terme (type jalons) qui intègrent ces effets dans l'espace et le temps.

La répartition des sédiments superficiels de même que les successions verticales de faciès enregistrées dans les carottes montrent une diminution globale d'énergie d'Est en Ouest, cohérente avec l'atténuation des courants de marée et de l'agitation, alors qu'inversement l'amplitude des remaniements est moins marquée à l'Est qu'à l'Ouest.

A l'Ouest, les enregistrements sédimentaires (dépôt/préservation drapages vaseux) matérialisent l'influence prépondérante de la dynamique tidale de relativement faible énergie avec des courants de marée de faible amplitude et de type giratoire. Cependant l'action du vent semble importante dans les processus de remaniements des sédiments fins superficiels, gorgés d'eau. En effet, un déplacement des nappes de vases vers la baie de Cancale s'observe par vents de secteurs Est - Sud-Est et inversement, les nappes se déplacent vers Hirel par vents de secteurs Ouest - Nord-Ouest. Par ailleurs, les érosions sur l'estran de Vildé-Hirel lors de périodes de vents de secteur Sud - Sud-Est sont encore plus nettes lorsqu'elles sont conjuguées avec des marées de vive-eau.

Au centre de la Baie, à Cherrueix, les faciès majoritairement sableux sont mis en place dans des conditions de plus forte hydrodynamique liée aux courants de marée plus forts et de type alternatif, combinés à l'action des houles. En dépit de cette forte énergie, la résultante des remaniements reste faible et moins importante qu'à Cancale et/ou à Vildé-Hirel où les sédiments sont plus vaseux et donc plus facilement mobilisables.

Des mesures de courant de plusieurs semaines ont permis de montrer la grande variabilité des courants sur l'estran en morte eau due à leur forte sensibilité au vent. En vive eau sur l'estran et à toutes périodes dans le reste du domaine, les courants sont plus réguliers. Le modèle SiAM reproduit la forte variabilité sur estran, et permet de simuler des amplitudes comparables aux mesures.

La comparaison des résultats obtenus à diverses échelles de temps par des mesures "in situ" et la modélisation hydrosédimentaire a permis de mettre en évidence l'importance de l'action du vent dans les remaniements sédimentaires, surtout sous une faible épaisseur de la tranche d'eau (morte-eau). Cette relation est cependant complexe, des vents de même secteur n'engendrant pas obligatoirement des évolutions similaires. Ces résultats soulignent les difficultés de quantification des remaniements sédimentaires superficiels des vases sur les vastes replats de marée évoluant en régime macrotidal. 


\section{Références}

Bonnot-Courtois C., Bassoullet P., Le Hir P., Tessier B., Cayocca F., Baltzer A. « Evolution et mobilité des sédiments superficiels de l'estran occidental de la Baie du Mont Saint Michel. » Actes IXèmes Journées Génie Côtier - Génie Civil, Brest, 2006, Session Dynamique sédimentaire et transport des particules. p. 57-64.

Bonnot-Courtois C., Caline B., L’Homer A. \& Le Vot M. La Baie du Mont-Saint-Michel et l'estuaire de la Rance. Environnements sédimentaires, aménagements et évolution récente. [The bay of Mont-Saint-Michel and the Rance estuary. Recent development and evolution of depositional environments]. - Bull. Centre Rech. Elf Explor. Prod., Mémoire n²6, 2002, 256 pp. TotalFinalElf - CNRS- EPHE ed.

Bonnot-Courtois C., Fournier J., Dreau A. "Recent morphodynamics of shell banks in the western part of the Bay of Mont Saint-Michel.” - Géomorphologie : relief, processus, environnement, 1, 2004, p. 65-80.

Caline B. Le secteur occidental de la Baie du Mont Saint Michel. Morphologie, sédimentologie et cartographie de l'estran à 1/20 000. - Documents du BRGM, nº 42, 1982, 250 pp.

Canuet F. Analyse des faciès sédimentaires dans le secteur occidental de la baie du Mont Saint-Michel. Mémoire Maîtrise. Univ. Caen, 2003, 15 pp.

Cayocca F., Dussauze M., Le Hir P., Bassoullet P., Jestin H. «Modélisation hydrosédimentaire de la baie du Mont Saint-Michel» » Actes IXèmes Journées Génie Côtier - Génie Civil, Brest, 2006, Session Dynamique sédimentaire et transport des particules. p. 65-74.

Ehrhold A. Dynamique de comblement d'un bassin sédimentaire soumis à un régime mégatidal : Exemple de la Baie du Mont Saint Michel, Thèse Université de Caen, 1999, 294 pp.

Jestin H., Le Hir P., Bassoullet P. "The SAMPLE system : a new concept of benthic station.” Proceedings of the Oceans' 94 Conference, IEEE, Brest, vol. III, 1994, p. 278-283.

Jestin H., Bassoullet P., Le Hir P., L'Yavanc J., Degres Y. “Development of ALTUS, a high frequency acoustic submersible recording altimeter to accurately monitor bed elevation and quantify deposition or erosion of sediments”. Oceans'98-IEEC/OES Conference. Nice, Conference Proceedings, vol. 1/3, 1998, p.189-194.

Larsonneur C. et coll. La Baie du Mont-Saint-Michel. - Bull. Inst. Géol. Bassin Aquit., vol. 46, 1989, p. 1-75.

Le Hir P., Cayocca F. "3D application of the continuous modelling concept to mud slides in open seas". J.W.C. Kranenburg (Editor), Fine Sediment Dynamics in the Marine Environment. Proceedings in Marine Science Elsevier, n5, 2002, p. 545-562.

Le Roy R., Simon B. Réalisation et validation d'un modèle de marée en Manche et dans le Golfe de Gascogne; Application à la réalisation d'un nouveau programme de réduction des sondages bathymétriques. Rapport d'études SHOM 2003. 


\section{REGC - x/2007. Génie Côtier}

L’Homer A., Courbouleix S., Beurrier M., Bonnot-Courtois C., Caline B., Ehrhold A., Lautridou J.P., Le Rhun J., Siméon Y., Thomas, Y. \& Villet M.. - Carte géologique France (1/50 000), feuille Baie du Mont-Saint-Michel (208). Orléans : BRGM. 1999 + Notice explicative, $184 \mathrm{pp}$.

Migniot C. Synthèse des connaissances hydro-sédimentaires de la baie du Mont Saint-Michel. Rapport DDE Manche/Mission Mont Saint-Michel - Syndicat Mixte pour le rétablissement du caractère maritime du Mont Saint-Michel. 1998, 111 pp. 